\title{
A support vector machine classifier for the prediction of osteosarcoma metastasis with high accuracy
}

\author{
YUNFEI HE ${ }^{1,2^{*}}$, JUN MA $^{1 *}$ and XIAOJIAN YE ${ }^{1}$ \\ ${ }^{1}$ Department of Orthopaedics, Changzheng Hospital Affiliated to Second Military Medical University, \\ Shanghai 200003; ${ }^{2}$ Department of Orthopaedics, Lanzhou General Hospital of \\ Lanzhou Military Command Region, Lanzhou, Gansu 730050, P.R. China
}

Received August 25, 2016; Accepted August 29, 2017

DOI: 10.3892/ijmm.2017.3126

\begin{abstract}
In this study, gene expression profiles of osteosarcoma (OS) were analyzed to identify critical genes associated with metastasis. Five gene expression datasets were screened and downloaded from Gene Expression Omnibus (GEO). Following assessment by MetaQC, the dataset GSE9508 was excluded for poor quality. Subsequently, differentially expressed genes (DEGs) between metastatic and non-metastatic OS were identified using meta-analysis. A protein-protein interaction (PPI) network was constructed with information from Human Protein Reference Database (HPRD) for the DEGs. Betweenness centrality (BC) was calculated for each node in the network and top featured genes ranked by $\mathrm{BC}$ were selected out to construct support vector machine (SVM) classifier using the training set GSE21257, which was then validated using the other three independent datasets. Pathway enrichment analysis was performed for the featured genes using Fisher's exact test. A total of 353 DEGs were identified and a PPI network including 164 nodes and 272 edges was then constructed. The top 64 featured genes ranked by BC were included in the SVM classifier. The SVM classifier exhibited high prediction accuracies in all of the 4 datasets, with accuracies of 100, 100, 92.6 and $100 \%$, respectively. Further analysis of the featured genes revealed that 11 Gene Ontology (GO) biological pathways and 5 Kyoto Encyclopedia of Genes and Genomes (KEGG) pathways were significantly over-represented, including the regulation of cell proliferation, regulation of apoptosis, pathways in cancer, regulation of actin cytoskeleton and the TGF- $\beta$ signaling pathway. On the whole, an SVM classifier with high prediction accuracy was constructed and validated, in which key genes associated with metastasis in OS were also revealed.
\end{abstract}

Correspondence to: Dr Xiaojian Ye, Department of Orthopaedics, Changzheng Hospital Affiliated to Second Military Medical University, 415 Fengyang Road, Shanghai 200003, P.R. China

E-mail: xjye2012@126.com

${ }^{*}$ Co-first authorship

Key words: osteosarcoma, metastasis, differentially expressed gene, protein-protein interaction network, support vector machine classifier
These findings may promote the development of genetic diagnostic methods and may enhance our understanding of the molecular mechanisms underlying the metastasis of OS.

\section{Introduction}

Osteosarcoma (OS) is most prevalent in children and young adults, and it is the 8th most common form of childhood cancer (1). Despite the success of chemotherapy for OS, it is still associated wiht one of the lowest survival rates for pediatric cancer. Even after the complete removal of the primary tumor, the metastasis of OS contributes to the poor prognosis of most patients.

With the progress being made in the elucidation of the molecular mechanisms responsible for tumor development, many researchers have tried to unveil the molecular mechanisms underlying the metastasis of OS, which may provide novel therapeutic targets or diagnostic biomarkers. The PI3K/ Akt signaling pathway $(2,3)$ and NF- $\mathrm{KB}$ pathway $(4,5)$ have been implicated in the promotion of OS metastasis. Notch signaling has been identified to play a critical role in the invasion and metastasis of OS (6). The HIF-1 $\alpha /$ CXCR4 pathway has also been shown to promote the hypoxia-induced metastasis of human OS cells (7).

Several genes implicated in OS metastasis have been disclosed, such as ezrin $(8,9)$. It has been reported that $\beta 4$ integrin promotes OS metastasis and interacts with ezrin (10). Moreover, aldolase A (11), cyclooxygenase-2 (12), and insulin-like growth factor binding protein 5 (13) have been shown to be involved in the metastasis of OS. However, knowledge of the molecular mechanisms responsible for the development of OS is limited. There is still a lack of effective targeted therapies and tumor-related biomarkers. Nevertheless, current knowledge is insufficient to markedly improve the outcomes of patients with metastatic OS.

Gene expression profiling has been utilized to identify critical genes associated with metastasis (14-16), as well as to develop novel treatments (17). In order to identify novel and reliable genes associated with metastasis, a meta-analysis of gene expression profiles was performed in the present study. Differentially expressed genes (DEGs) between metastatic and non-metastatic OS were revealed, followed by protein-protein interaction (PPI) network analysis. The featured genes were 
disclosed via support vector machine (SVM) classification. Moreover, an SVM classifier was acquired and validated.

\section{Materials and methods}

Gene expression profiles and pre-treament. Gene expression datasets were retrieved from Gene Expression Omnibus (GEO) using the key words 'osteosarcoma', 'metastasis', and 'Homo sapiens' by the end of April 28th, 2016. Five datasets (Table I) that met the following criteria were collected: i) gene expression data; ii) OS; and iii) information about metastasis was described.

The gene expression datasets GSE14359 and GSE14827 were acquired with Affymetrix platforms. Thus, background correction and normalization were performed with package 'affy' of R. Missing values were filled with the median method. Background correction was carried out using the MSA method. Normalization was achieved with the quantiles method.

For the other 3 gene expression datasets, probes were then mapped into genes. Probes corresponding to the same gene were averaged as the final expression value of the gene. Normalization was performed with package limma (18) of R.

Screening of DEGs. Prior to meta-analysis, the 5 gene expression datasets were assessed using package MetaQC (19), as well as principal component analysis (PCA) and standardized mean rank (SMR). The following criteria were applied in the assessment: i) internal quality control (IQC), homogeneity test of gene expression profiles among datasets; ii) external quality control (EQC), homogeneity test of gene expression profiles with pathway database; iii) accuracy quality control (AQC), accuracy of featured genes (AQCg) or pathways (AQCp); and iv) consistency quality control (CQC), consistency in ranking of featured genes (CQCg) or pathways (CQCp).

DEGs were screened out using the MetaDE.ES from package MetaDE. This method first tested the heterogeneity of gene expression value in various platforms with three statistic parameters: $\tau^{2}$, Q-value and Qpval. It then tested differential expression of genes between different groups with statistic parameters P-value. To ensure the homogeneity of featured genes, $\tau^{2}=0$, Qpval $>0.05$ and $\mathrm{P}<0.05$ were set as the cut-offs.

Construction of protein-protein interaction (PPI) network. The PPI information was downloaded from the database of protein, chemical, and genetic interactions (BioGRID, http://thebiogrid.org/) (20), Human Protein Reference Database (HPRD, http://www.hprd.org/) (21) and Database of Interacting Proteins (DIP, http://dip.doe-mbi.ucla. edu/dip/Main.cgi) (22). The protein products of the DEGs were mapped into the whole network and the PPI network for the DEGs was then acquired. The network was then visualized with Cytoscape (23).

Calculation of betweenness centrality $(B C)$. The featured genes were screened out from the DEGs using $\mathrm{BC}$, which reflected hubness of the node in the PPI network. The BC was calculated as follows:

$$
\mathrm{C}_{\mathrm{B}}(v)=\sum_{t \neq v \neq u \in V} \frac{\sigma_{s t}(v)}{\sigma_{s t}}
$$

where $\sigma_{s t}$ is the shortest path from $s$ to $t ; \sigma_{s t}(v)$ is the number of the shortest path from $s$ to $t$ passing through node $V$; BC score is between 0 and 1 , and the greater $\mathrm{BC}$ score indicates the higher degree of hubness.

Training and validation of SVM classifier. The dataset GSE21257 was selected as the training set. Genes were ranked based upon the BC value and top 10 genes were selected out to train the SVM classifier. An increment of 10 genes were added into the classifier until the metastatic OS could be totally separated from non-metastatic OS. These DEGs were regarded as featured genes. Two-way clustering of sample and gene expression was applied on the featured genes and the result was visualized with a heatmap. The other 3 gene expression datasets were used as the validation set. Sensitivity (Se), specificity $(\mathrm{Sp})$, positive predictive value (PPV), negative predictive value (NPV) and the area under the curve (AUC) were calculated to evaluate the SVM classifier.

Pathway enrichment analysis. Gene Ontology (GO) biological pathways and Kyoto Encyclopedia of Genes and Genomes (KEGG) pathways related to featured genes were identified using runHyperGO and runHyperKEGG from package EMA of R. The significance was calculated using Fisher's exact test as follows:

$$
\mathrm{p}=1-\sum_{i=0}^{x-1} \frac{\left(\begin{array}{c}
\mathrm{M} \\
\mathrm{i}
\end{array}\right)\left(\begin{array}{l}
\mathrm{N}-\mathrm{M} \\
\mathrm{K}-\mathrm{i}
\end{array}\right)}{\left(\begin{array}{l}
\mathrm{N} \\
\mathrm{K}
\end{array}\right)}
$$

where $\mathrm{N}$ is the total number of gene; $\mathrm{M}$ is the number of gene in the pathway; $\mathrm{K}$ is the number of featured gene.

\section{Results}

DEGs. Quality control test was applied on the 5 gene expression datasets and the results are listed in Table II. As shown in the PCA result (Fig. 1), the dataset GSE9508 far deviated from the other 4 datasets, which was assessed as poor quality. Thus, the dataset GSE9508 was excluded from the following analysis.

According to the criteria described above, a total of 353 DEGs were identified from the 4 datasets. The top 10 DEGs (ranked by P-value) are listed in Table III, including chromosome 13 open reading frame 18 (C13orf18), activating transcription factor 3 (ATF3), cadherin 5 ( $\mathrm{CDH} 5)$, epoxide hydrolase 2 (EPHX2), cadherin 3 ( $\mathrm{CDH} 3)$, ectonucleoside triphosphate diphosphohydrolase 5 (ENTPD5), forkhead box A1 (FOXA1), ephrin B2 (EFNB2), bromo adjacent homology domain containing $1(B A H D I)$ and chromosome 10 open reading frame 76 (C10orf76).

PPI network. Non-DEGs interacting with no less than 5 DEGs were also included in the PPI network. Finally, a PPI network containing 164 nodes and 272 edges was obtained (Fig. 2). The distribution of degree is shown in Fig. 3. Most genes (168 genes) showed a small degree (Log transformed degree $<1$ ), while only 2 genes had a Log transformed degree of $>4$. Therefore, similar to most biological networks, this PPI network exhibited 
Table I. Information of the gene expression datasets from GEO.

\begin{tabular}{llcccc}
\hline GEO accession & Platform & Probe no. & Total sample no. & Metastasis & Non-metastasis \\
\hline GSE32981 & GPL3307 & 14725 & 23 & 18 & 5 \\
GSE21257 & GPL10295 & 48701 & 53 & 34 & 8 \\
GSE14359 & GPL96 & 22283 & 18 & 9 & 10 \\
GSE14827 & GPL570 & 42450 & 27 & 21 & 18 \\
GSE9508 & GPL6067 & 41059 & 34 & 13 \\
\hline
\end{tabular}

GEO, Gene Expression Omnibus.

Table II. Quality control test of the gene expression datasets.

\begin{tabular}{lllccrrr}
\hline Dataset & IQC & EQC & CQCg & CQCp & AQCg & AQCp & SMR \\
\hline GSE32981 & 5.27 & 3.23 & 106.65 & 158.86 & 32.71 & 90.88 & 1.62 \\
GSE21257 & 4.38 & 3.16 & 64.14 & 146.51 & 26.46 & 96.74 & 2.86 \\
GSE14359 & 4.81 & 3.23 & 59.25 & 171.49 & 25.5 & 84.37 & 2.42 \\
GSE14827 & 6.09 & 1.1 & 101.1 & 114.3 & 19.53 & 29.46 \\
GSE9508 & 0.23 & 1.69 & 6.01 & 1.58 & 1.26 & 0.71 & 11.58 \\
\hline
\end{tabular}

IQC, internal quality control; EQC, external quality control; CQCg, consistency quality control of genes; CQCp, consistency quality control of pathways; AQCg, accuracy quality control of genes; AQCp, accuracy quality control of pathways; SMR, standardized mean rank.

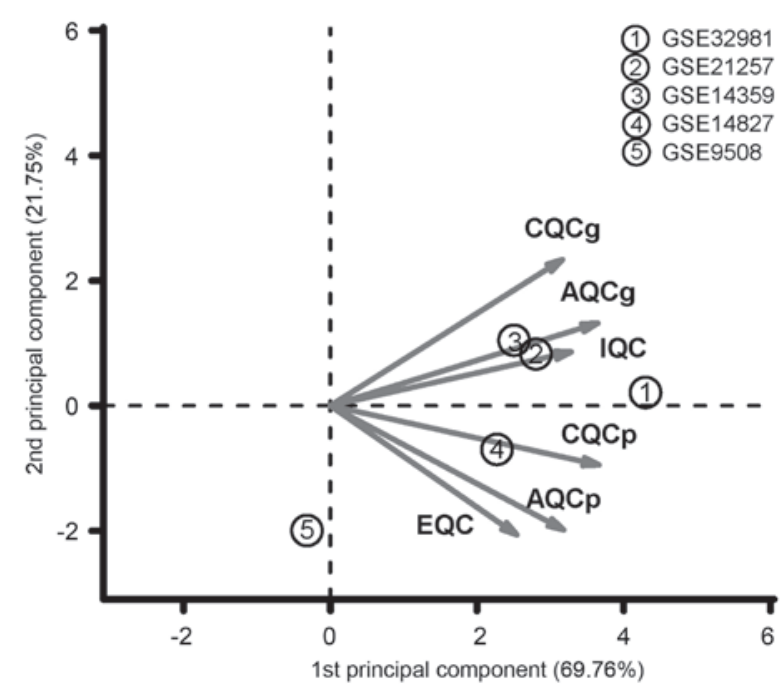

Figure 1. PCA results of the 5 gene expression datasets. PCA, principal component analysis; $\mathrm{CQCg}$, consistency quality control of genes; $\mathrm{AQCg}$, accuracy quality control of genes; IQC, internal quality control; CQCp, consistency quality control of pathways; AQCp, accuracy quality control of pathways; EQC, external quality control.

scale-free property. These genes with a high degree were hub genes and may thus play important roles in the development of diseases.

Featured genes. BC was calculated for each node and the top 10 genes are listed in Table IV. These were estrogen receptor 1 (ESRI), E1A binding protein p300 (EP300), tumor protein p53 (TP53), SMAD family member 3 (SMAD3), epidermal growth factor receptor $(E G F R)$, fibronectin $1(F N 1)$, SRC proto-oncogene, non-receptor tyrosine kinase $(S R C)$, protein kinase $\mathrm{C} \alpha(P R K C A)$, DEAD-box helicase 5 (DDX5) and erythropoietin receptor $(E P O R)$.

SVM classifier. Top genes were selected out to train the SVM classifier with the dataset GSE21257. As shown in Fig. 4A, the accuracy increased from 75 to $100 \%$, while more genes were included in the classifier. The accuracy reached $100 \%$, while the top 100 genes (containing 64 DEGs; data not shown) were included in the classifier. Therefore, the top 64 featured genes (data not shown) were selected to construct the SVM classifier. The classifier could separate metastatic OS from non-metastatic OS in the dataset GSE21257 (Fig. 4B).

Hierarchical clustering was performed for the 53 samples from the training dataset GSE21257 using the 64 featured genes and the results are shown in Fig. 5. The 64 featured genes could well distinguish metastatic OS samples from non-metastatic OS samples.

The SVM classifier was validated using the other 3 independent datasets GSE32981, GSE14827 and GSE14359. The accuracies were high in all datasets, with a prediction accuracy of $100 \%$ in dataset GSE32981 (Fig. 6A) and GSE14359 (Fig. 6C) and 92.6\% in dataset GSE14827 (Fig. 6B). Two non-metastatic OS samples were predicted wrong as metastatic samples in dataset GSE14827. The accuracy, sensitivity, specificity, PPV, NPV and AUC are listed in Table V.

Over-represented biological pathways. A total of $11 \mathrm{GO}$ biological pathways (Fig. 7) and 5 KEGG pathways (Table VI) were identified in the 64 featured genes. Some pathways were 
Table III. Top 10 DEGs ranked by P-value.

\begin{tabular}{|c|c|c|c|c|c|}
\hline Gene & P-value & Qval & Qpval & $\tau \operatorname{tau}^{2}$ & Expression \\
\hline C13orf18 & $3.20 \mathrm{E}-05$ & 8.32E-01 & 8.42E-01 & 0 & + \\
\hline$A T F 3$ & $6.41 \mathrm{E}-05$ & $1.02 \mathrm{E}+00$ & 7.97E-01 & 0 & + \\
\hline CDH5 & $6.41 \mathrm{E}-05$ & $1.82 \mathrm{E}+00$ & $6.11 \mathrm{E}-01$ & 0 & + \\
\hline EPHX2 & $6.41 \mathrm{E}-05$ & $1.68 \mathrm{E}+00$ & $6.42 \mathrm{E}-01$ & 0 & - \\
\hline $\mathrm{CDH3}$ & $1.25 \mathrm{E}-04$ & $2.64 \mathrm{E}+00$ & $4.51 \mathrm{E}-01$ & 0 & + \\
\hline ENTPD5 & $1.64 \mathrm{E}-04$ & $1.77 \mathrm{E}+00$ & $6.21 \mathrm{E}-01$ & 0 & - \\
\hline FOXAI & $1.67 \mathrm{E}-04$ & $1.80 \mathrm{E}+00$ & $6.16 \mathrm{E}-01$ & 0 & + \\
\hline EFNB2 & $1.81 \mathrm{E}-04$ & $2.84 \mathrm{E}+00$ & 4.17E-01 & 0 & + \\
\hline BAHDI & $2.10 \mathrm{E}-04$ & $1.01 \mathrm{E}+00$ & 7.99E-01 & 0 & - \\
\hline C10orf76 & $2.38 \mathrm{E}-04$ & $2.96 \mathrm{E}+00$ & 3.98E-01 & 0 & - \\
\hline
\end{tabular}

'+' in the column 'Expression' indicates upregulation in metastatic OS compared with non-metastatic OS, while '-' indicates downregulation in metastatic OS. DEGs, differentially expressed genes; OS, osteosarcoma.

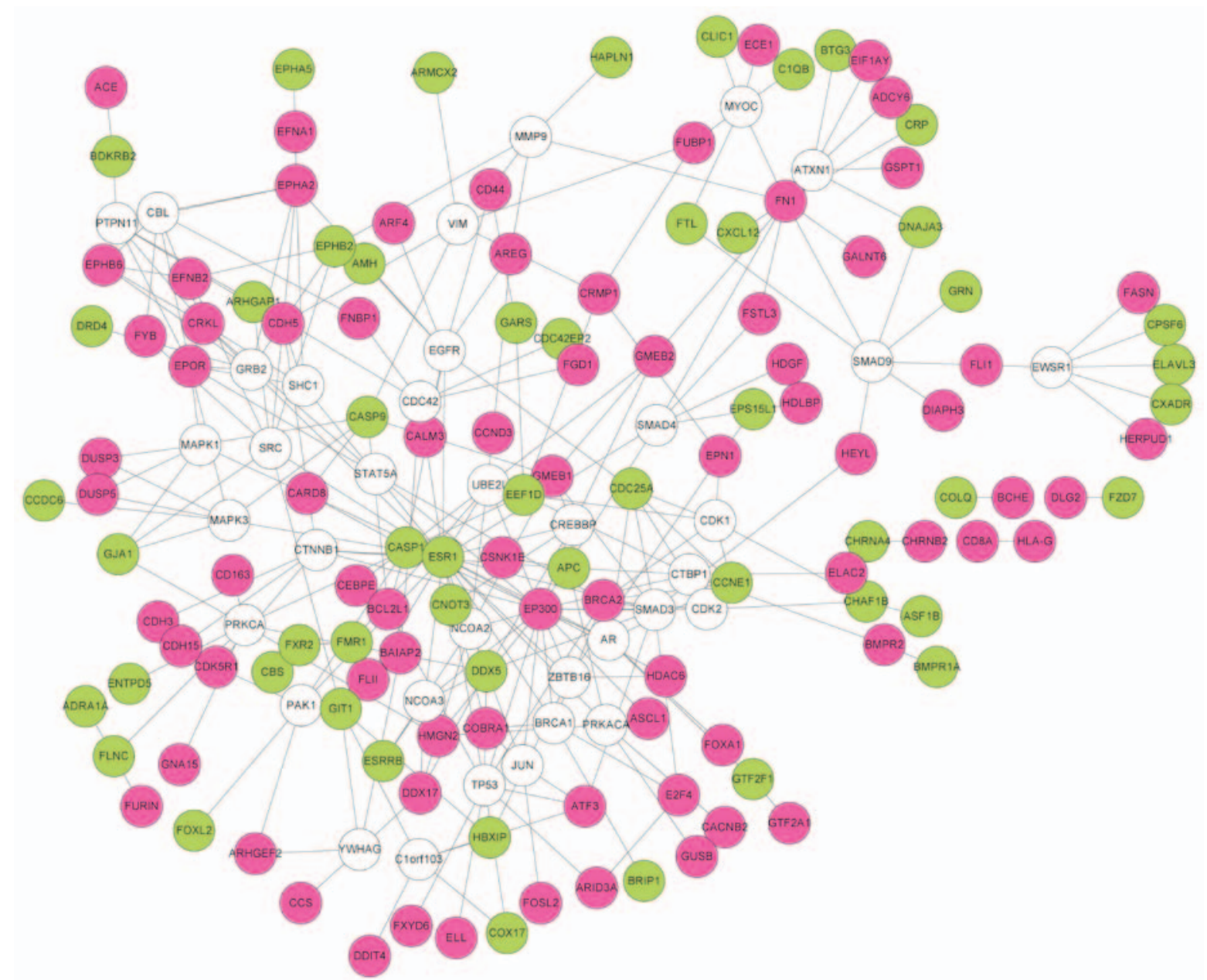

Figure 2. PPI network of the DEGs. Upregulated genes in metastatic OS are shown in red, downregulated genes in metastatic OS are shown in green and nonDEGs are shown in white. PPI, protein-protein interaction; DEGs, differentially expressed genes; OS, osteosarcoma.

significantly over-represented in the featured genes, such as regulation of cell proliferation, regulation of apoptosis, pathways in cancer, regulation of actin cytoskeleton and the TGF- $\beta$ signaling pathway.

\section{Discussion}

Four gene expression datasets were included in this meta-analysis. A total of 353 DEGs were identified and a PPI network including 
Table IV. Top 10 genes ranked by BC.

\begin{tabular}{lcccccr}
\hline Gene & BC & EXP & Degree & P-value & Qval & Qpval \\
\hline ESR1 & $4.03 \mathrm{E}-01$ & - & 26 & $1.60 \mathrm{E}-02$ & $2.73 \mathrm{E}+00$ & $4.35 \mathrm{E}-01$ \\
EP300 & $1.90 \mathrm{E}-01$ & + & 19 & $4.86 \mathrm{E}-02$ & $1.12 \mathrm{E}+00$ & $7.73 \mathrm{E}-01$ \\
TP53 & $6.70 \mathrm{E}-02$ & Null & 10 & - & - & - \\
SMAD3 & $1.02 \mathrm{E}-01$ & Null & 9 & - & - & - \\
EGFR & $7.40 \mathrm{E}-02$ & Null & 9 & - & - & - \\
FN1 & $1.33 \mathrm{E}-01$ & + & 8 & $9.80 \mathrm{E}-03$ & $2.63 \mathrm{E}+00$ & - \\
SRC & $9.92 \mathrm{E}-02$ & Null & 8 & - & - & - \\
PRKCA & $8.43 \mathrm{E}-02$ & Null & 8 & - & - & - \\
DDX5 & $6.33 \mathrm{E}-02$ & - & 8 & $1.00 \mathrm{E}-03$ & $1.04 \mathrm{E}+00$ & - \\
EPOR & $2.35 \mathrm{E}-02$ & + & 8 & $3.80 \mathrm{E}-02$ & - & - \\
\hline
\end{tabular}

' + ' in the column 'EXP' indicates upregulation in metastatic OS, while '-' indicates downregulation and 'null' indicates no differential expression. BC, betweenness centrality; OS, osteosarcoma.

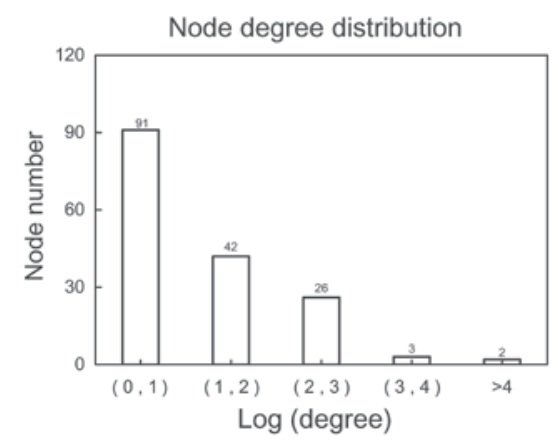

Figure 3. Distribution of degree in the PPI network. The $\mathrm{x}$-axis is Log transformed degree and $y$-axis is number of node. PPI, protein-protein interaction.

164 nodes, as well as 272 edges was constructed. The top 64 featured genes ranked by BC were included in the SVM classifier. The prediction accuracies for the 4 datasets were 100 , $100,92.6$ and $100 \%$, respectively. Pathways, such as regulation of cell proliferation, regulation of apoptosis, pathways in cancer, regulation of actin cytoskeleton and the TGF- $\beta$ signaling pathway were significantly over-represented in the 64 featured genes. These pathways were closely associated with cancer and cell mobility.

In the featured genes, some have been implicated in the metastasis of OS. CD44 molecule $(C D 44)$ is a cell-surface glycoprotein involved in cell-cell interactions, cell adhesion and migration. It participates in a wide variety of cellular functions, including metastasis. Kim et al reported that the expression of CD44 isoforms correlated with the metastatic potential of OS (24). Zhao et al found that miR-34a inhibited the metastasis of OS cells by suppressing the expression of CD44 (25). $\mathrm{EPH}$ receptor $\mathrm{A} 2$ (EPHA2) is involved in the mitogenic signaling pathway and it may stimulate OS metastasis (26). EPHA2 was identified as a receptor for the targeted drug delivery of OS (27). High mobility group nucleosomal binding domain 2 (HMGN2) exhibits inhibitory effects on growth and migration of human OS cell lines (28). Yang et al found that recurrent LDL receptor-related protein 1 (LRPI)-small nuclear ribonucleoprotein U11/U12 subunit 25 (SNRNP25)
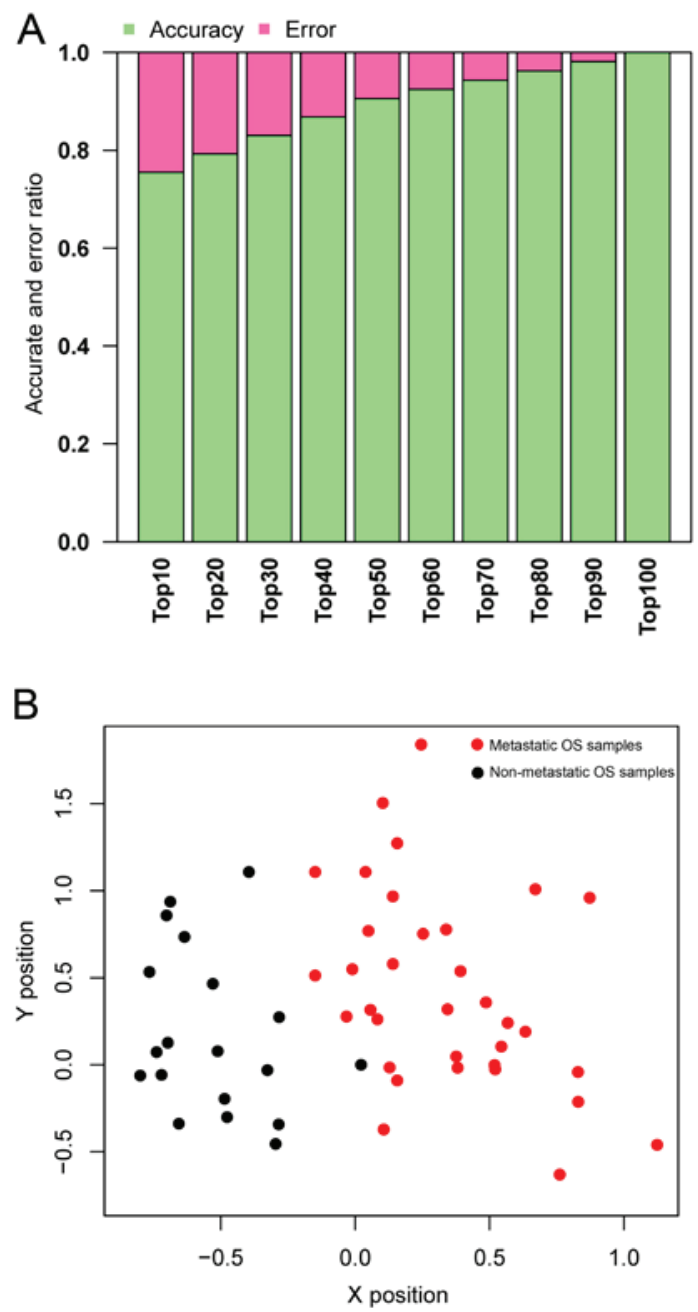

Figure 4. (A) Predictive accuracy with different numbers of featured genes Accuracy is indicated in green, while error rate is indicated in red. (B) Scatter plots describing prediction results of dataset GSE21257. Metastatic OS samples are in red while non-metastatic OS samples are in black. OS, osteosarcoma.

and potassium calcium-activated channel subfamily $\mathrm{M}$ regulatory $\beta$ subunit 4 (KCNMB4)-cyclin D3 (CCND3) fusion genes promoted tumor cell motility in human OS (29). The 


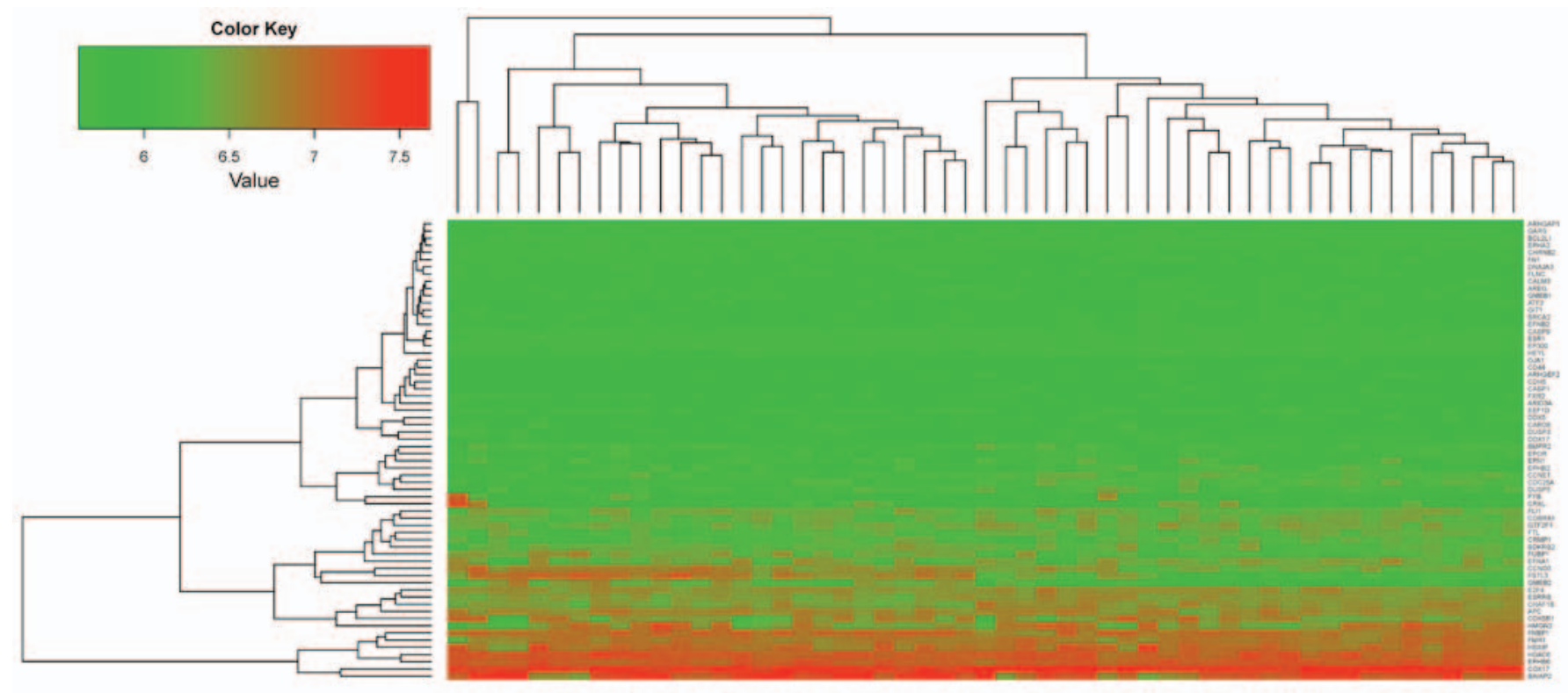

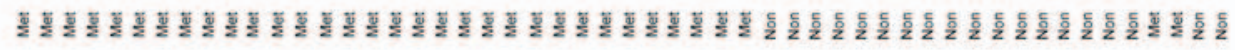

Figure 5. Hierarchical clustering results of OS samples using the 64 featured genes. OS, osteosarcoma.
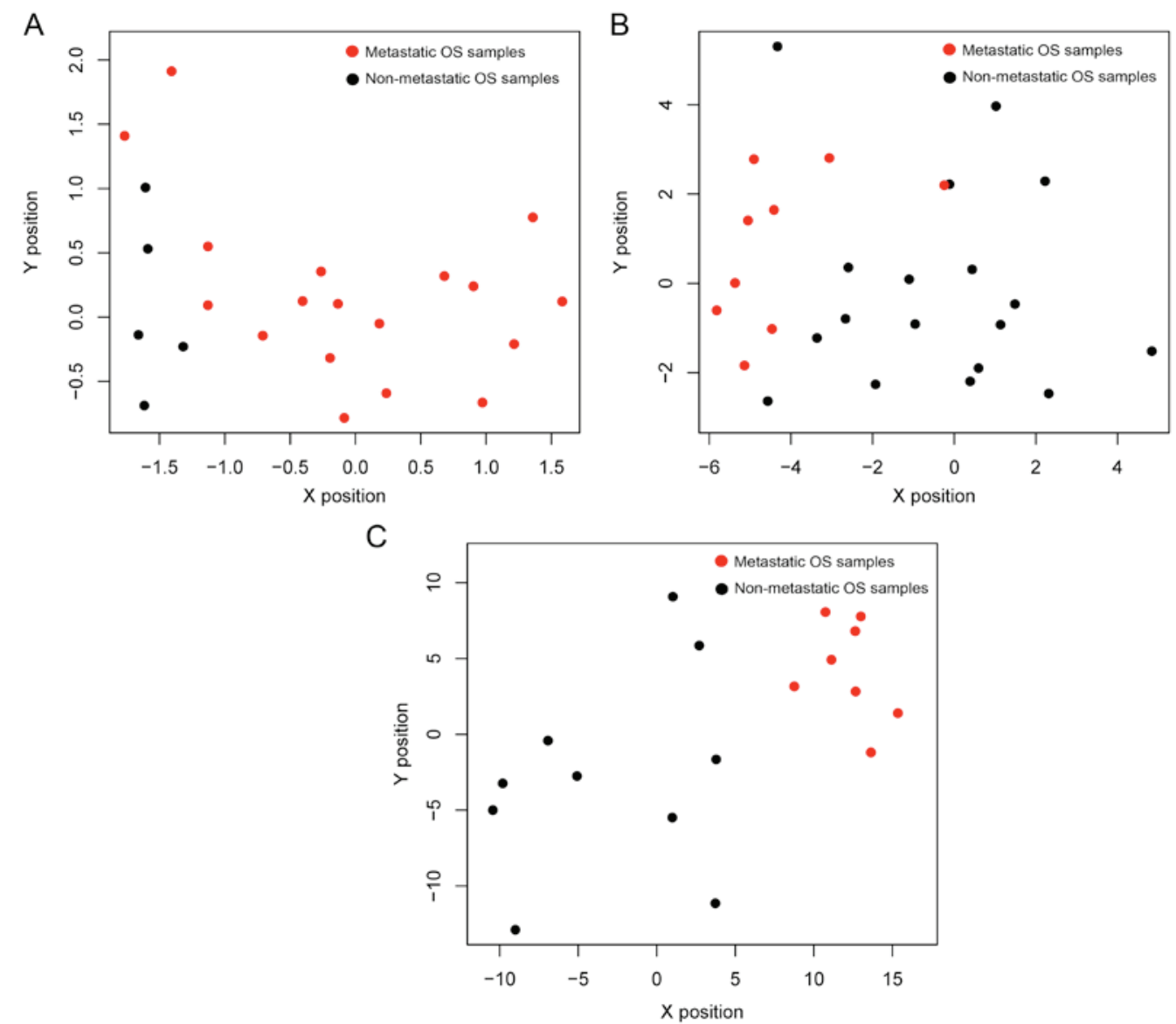

Figure 6. Scatter plots describing the prediction results of datasets (A) GSE32981, (B) GSE14827 and (C) GSE14359 using the SVM classifier. Metastatic OS samples are shown in red, while non-metastatic OS samples are shown in black. SVM, support vector machine; OS, osteosarcoma.

overexpression of cyclin E1 (CCNEI) has been observed in many tumors. The inhibition of $C C N E 1$ suppresses the proliferation of OS cells (30).

Several featured genes were not reported in the metastasis of OS, while they have been previously shown to be involved in the metastasis of other types of cancer. Histone deacetylase 6 (HDAC6) has been shown to be involved in the metastasis of melanoma cells (31) and oral squamous cell carcinoma (OSCC) cells (32). The reduced expression of $\mathrm{EPH}$ receptor $\mathrm{B} 2$ (EPHB2) parallels invasion and metastasis in 
Table V. Prediction results of the SVM classifier.

\begin{tabular}{|c|c|c|c|c|c|c|c|c|}
\hline Datasets & $\begin{array}{c}\text { No. of } \\
\text { samples }\end{array}$ & $\begin{array}{l}\text { Prediction } \\
\text { error }\end{array}$ & Accuracy & Sensitivity & Specificity & PPV & NPV & AUROC \\
\hline GSE21257 & 53 & 0 & 1.000 & 1.000 & 1.000 & 1.000 & 1.000 & 1.000 \\
\hline GSE32981 & 23 & 0 & 1.000 & 1.000 & 1.000 & 1.000 & 1.000 & 1.000 \\
\hline GSE14827 & 27 & 2 & 0.926 & 1.000 & 0.889 & 0.818 & 1.000 & 0.931 \\
\hline GSE14359 & 18 & 0 & 1.000 & 1.000 & 1.000 & 1.000 & 1.000 & 1.000 \\
\hline
\end{tabular}

SVM, support vector machine; PPV, positive predictive value; NPV, negative predictive value; AUROC, area under the ROC curve.

Table VI. KEGG pathways significantly over-represented in the 64 featured genes.

\begin{tabular}{lll}
\hline KEGG pathway & P-value & \multicolumn{1}{c}{ Genes } \\
\hline hsa05200:pathways in cancer & $1.28 \mathrm{E}-02$ & $C C N E 1, C R K L, E P 300, C A S P 9, B R C A 2, B C L 2 L 1, F N 1, A P C$ \\
hsa04110:cell cycle & $1.61 \mathrm{E}-02$ & $C C N E 1, E P 300, E 2 F 4, C C N D 3, C D C 25 A$ \\
hsa04360: axon guidance & $1.78 \mathrm{E}-02$ & EPHB6, EFNA1, EFNB2,EPHA2, EPHB2 \\
hsa04810:regulation of actin cytoskeleton & $2.54 \mathrm{E}-02$ & GIT1, CRKL, BAIAP2, BDKRB2, FN1, APC \\
hsa04350:TGF- $\beta$ signaling pathway & $3.03 \mathrm{E}-02$ & $A M H, E P 300, E 2 F 4, B M P R 2$ \\
\hline
\end{tabular}

KEGG, Kyoto Encyclopedia of Genes and Genomes.

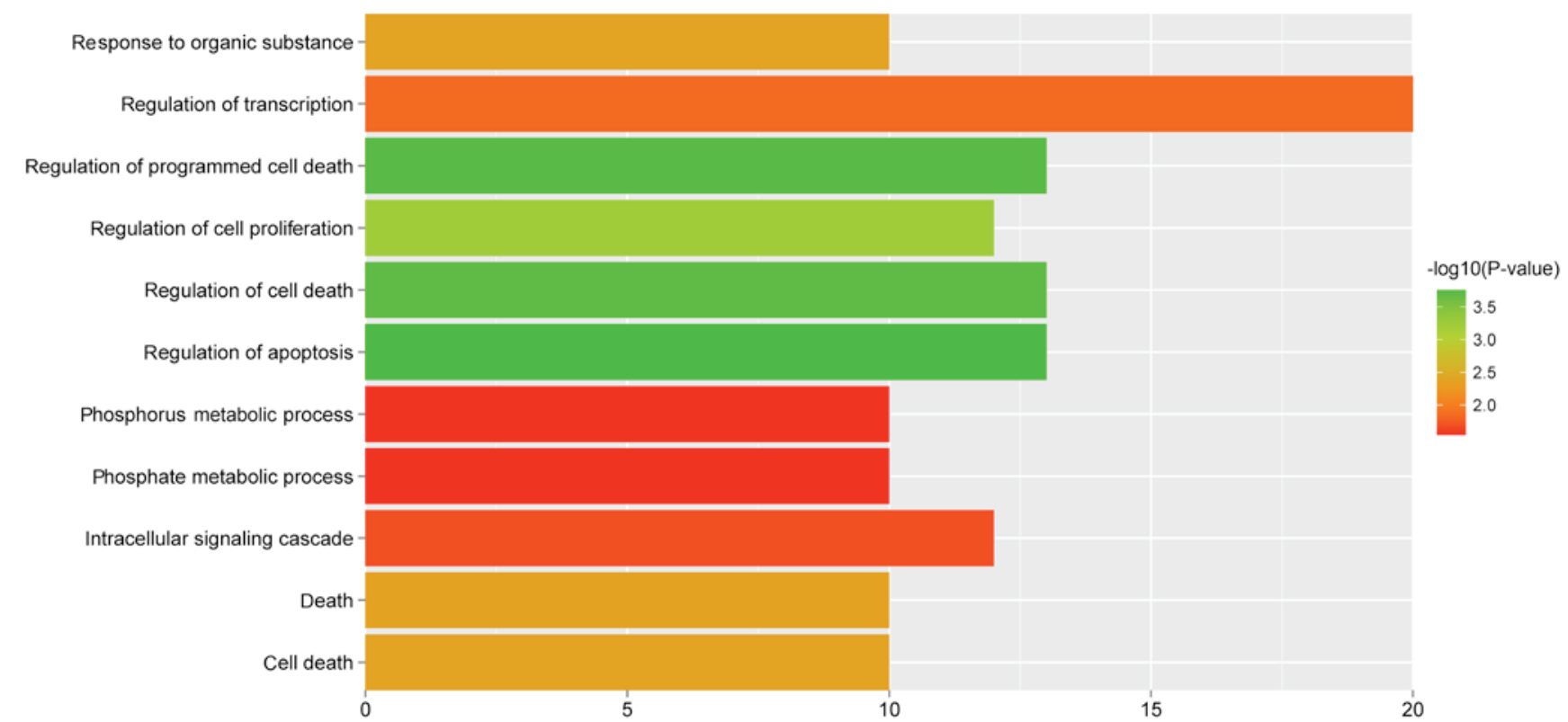

Figure 7. GO biological pathways significantly over-represented in the 64 featured genes. The x-axis indicates the number of genes and the color of the bar indicates the $-\log$ (P-value). GO, Gene Ontology.

colorectal tumors (33). The overexpression of the EPHA4 gene and the reduced expression of the $E P H B 2$ gene correlate with liver metastasis in colorectal cancer (34). Cell division cycle 25A $(C D C 25 A)$ regulates matrix metalloprotease 1 through Foxo1 and mediates the metastasis of breast cancer cells (35). Their roles in OS metastasis remain to be disclosed with further research.

Hypermethylated ESRI gene promoter in serum can be used as a candidate biomarker for the diagnosis and treatment efficacy of breast cancer metastasis (36). The aberrant DNA methylation of ESR1 may be a useful as prognostic indicator in OS (37). We speculated that it may be involved in OS metastasis. Further studies may provide potential diagnostic biomarkers or therapeutic targets for OS.

In conclusion, in this study, an SVM classifier of high accuracy was obtained with 64 featured genes. Several critical genes associated with OS metastasis were also revealed, some of which are not previously implicated in OS. The findings based on bioinformatics analysis should be further confirmed by experimental studies. 
These findings may promote the development of genetic diagnostic methods and may enhance our understanding of the molecular mechanisms underlying the metastais of OS.

\section{Acknowledgements}

This study was supported by the National Natural Science Foundation of China(Grant No. 81472071 and GrantNo. 81772445).

\section{References}

1. Jaffe N, Bruland OS and Bielack S (eds): Pediatric and Adolescent Osteosarcoma. Springer 152, Berlin, 2010.

2. Hou CH, Lin FL, Tong KB, Hou SM and Liu JF: Transforming growth factor alpha promotes osteosarcoma metastasis by ICAM-1 and PI3K/Akt signaling pathway. Biochem Pharmacol 89: 453-463, 2014.

3. Dong Y, Liang G, Yuan B, Yang C, Gao R and Zhou X: MALAT1 promotes the proliferation and metastasis of osteosarcoma cells by activating the PI3K/Akt pathway. Tumour Biol 36: 1477-1486, 2015.

4. Guo YS, Zhao R, Ma J, Cui W, Sun Z, Gao B, He S, Han YH, Fan J, Yang L, et al: ßig-h3 promotes human osteosarcoma cells metastasis by interacting with integrin $\alpha 2 \beta 1$ and activating PI3K signaling pathway. PLoS One 9: e90220, 2014.

5. Liao D, Zhong L, Duan T, Zhang RH, Wang X, Wang G, Hu K, Lv X and Kang T: Aspirin suppresses the growth and metastasis of osteosarcoma through the NF- $\kappa \mathrm{B}$ pathway. Clin Cancer Res 21: 5349-5359, 2015.

6. Zhang P, Yang Y, Zweidler-McKay P and Hughes DPM: Retraction: Critical role of notch signaling in osteosarcoma invasion and metastasis. Clin Cancer Res 19: 5256-5257, 2013.

7. Guan G, Zhang Y, Lu Y, Liu L, Shi D, Wen Y, Yang L, Ma Q, Liu T, Zhu X, et al: The HIF-1 $\alpha / C X C R 4$ pathway supports hypoxia-induced metastasis of human osteosarcoma cells. Cancer Lett 357: 254-264, 2015.

8. Khanna C, Wan X, Bose S, Cassaday R, Olomu O, Mendoza A, Yeung C, Gorlick R, Hewitt SM and Helman LJ: The membrane-cytoskeleton linker ezrin is necessary for osteosarcoma metastasis. Nat Med 10: 182-186, 2004.

9. Ren L, Hong SH, Cassavaugh J, Osborne T, Chou AJ, Kim SY, Gorlick R, Hewitt SM and Khanna C: The actin-cytoskeleton linker protein ezrin is regulated during osteosarcoma metastasis by PKC. Oncogene 28: 792-802, 2009.

10. Wan X, Kim SY, Guenther LM, Mendoza A, Briggs J, Yeung C, Currier D, Zhang H, Mackall C, Li WJ, et al: Beta4 integrin promotes osteosarcoma metastasis and interacts with ezrin. Oncogene 28: 3401-3411, 2009.

11. Long F, Cai X, Luo W, Chen L and Li K: Role of aldolase A in osteosarcoma progression and metastasis: In vitro and in vivo evidence. Oncol Rep 32: 2031-2037, 2014.

12. Qu L and Liu B: Cyclooxygeanse-2 promotes metastasis in osteosarcoma. Cancer Cell Int 15: 69, 2015.

13. Su Y, Wagner ER, Luo Q, Huang J, Chen L, He BC, Zuo GW, Shi Q, Zhang BQ, Zhu G, et al: Insulin-like growth factor binding protein 5 suppresses tumor growth and metastasis of human osteosarcoma. Oncogene 30: 3907-3917, 2011.

14. Namløs HM, Kresse SH, Müller CR, Henriksen J, Holdhus R, Sæter G, Bruland OS, Bjerkehagen B, Steen VM and Myklebost O Global gene expression profiling of human osteosarcomas reveals metastasis-associated chemokine pattern. Sarcoma 2012: 639038, 2012.

15. Odagiri H, Kadomatsu T, Endo M, Masuda T, Morioka MS, Fukuhara S, Miyamoto T, Kobayashi E, Miyata K, Aoi J, et al: The secreted protein ANGPTL2 promotes metastasis of osteosarcoma cells through integrin $\alpha_{5} \beta_{1}$, p38 MAPK, and matrix metalloproteinases. Sci Signal 7: ra7, 2014.

16. Endo-Munoz L, Cumming A, Rickwood D, Wilson D, Cueva C, $\mathrm{Ng} \mathrm{C}$, Strutton G, Cassady AI, Evdokiou A, Sommerville S, et al: Loss of osteoclasts contributes to development of osteosarcoma pulmonary metastases. Cancer Res 70: 7063-7072, 2010.

17. Buddingh EP, Kuijjer ML, Duim RA, Bürger H, Agelopoulos K, Myklebost O, Serra M, Mertens F, Hogendoorn PC, Lankester AC, et al: Tumor-infiltrating macrophages are associated with metastasis suppression in high-grade osteosarcoma: A rationale for treatment with macrophage activating agents. Clin Cancer Res 17: 2110-2119, 2011.
18. Smyth GK: Limma: Linear Models for Microarray Data. In: Bioinformatics and Computational Biology Solutions Using R and Bioconductor. Gentleman R, Carey VJ, Huber W, Irizarry RA, Dudoit S (eds). Springer New York, NY, pp397-420, 2005.

19. Wang X, Kang DD, Shen K, Song C, Lu S, Chang LC, Liao SG, Huo Z, Tang S, Ding Y, et al: An R package suite for microarray meta-analysis in quality control, differentially expressed gene analysis and pathway enrichment detection. Bioinformatics 28 : 2534-2536, 2012

20. Chatr-Aryamontri A, Breitkreutz BJ, Oughtred R, Boucher L, Heinicke S, Chen D, Stark C, Breitkreutz A, Kolas N, O'Donnell L, et al: The BioGRID interaction database: 2015 update. Nucleic Acids Res 43: D470-D478, 2015.

21. Keshava Prasad TS, Goel R, Kandasamy K, Keerthikumar S, Kumar S, Mathivanan S, Telikicherla D, Raju R, Shafreen B, Venugopal A, et al: Human Protein Reference Database -- 2009 update. Nucleic Acids Res 37: D767-D772, 2009.

22. Xenarios I, Rice DW, Salwinski L, Baron MK, Marcotte EM and Eisenberg D: DIP: The database of interacting proteins. Nucleic Acids Res 28: 289-291, 2000.

23. Smoot ME, Ono K, Ruscheinski J, Wang PL and Ideker T: Cytoscape 2.8: New features for data integration and network visualization. Bioinformatics 27: 431-432, 2011.

24. Kim HS, Park YB, Oh JH, Jeong J, Kim CJ and Lee SH: Expression of CD44 isoforms correlates with the metastatic potential of osteosarcoma. Clin Orthop Relat Res 396: 184-190, 2002.

25. Zhao H, Ma B, Wang Y, Han T, Zheng L, Sun C, Liu T, Zhang Y, Qiu X and Fan Q: miR-34a inhibits the metastasis of osteosarcoma cells by repressing the expression of CD44. Oncol Rep 29: 1027-1036, 2013.

26. Fritsche-Guenther R, Noske A, Ungethüm U,Kuban RJ, Schlag PM, Tunn PU, Karle J, Krenn V, Dietel M and Sers C: De novo expression of EphA2 in osteosarcoma modulates activation of the mitogenic signalling pathway. Histopathology 57: 836-850, 2010.

27. Posthumadeboer J, Piersma SR, Pham TV, van Egmond PW, Knol JC, Cleton-Jansen AM, van Geer MA, van Beusechem VW, Kaspers GJ, van Royen BJ, et al: Surface proteomic analysis of osteosarcoma identifies EPHA2 as receptor for targeted drug delivery. Br J Cancer 109: 2142-2154, 2013.

28. Liang G, Xu E, Yang C, Zhang C, Sheng $X$ and Zhou X: Nucleosome-binding protein HMGN2 exhibits antitumor activity in human $\mathrm{SaO} 2$ and $\mathrm{U} 2-\mathrm{OS}$ osteosarcoma cell lines. Oncol Rep 33: 1300-1306, 2015.

29. Yang J, Annala M, Ji P, Wang G, Zheng H, Codgell D, Du X, Fang Z, Sun B, Nykter M, et al: Recurrent LRPI-SNRNP25 and $K C N M B 4-C C N D 3$ fusion genes promote tumor cell motility in human osteosarcoma. J Hematol Oncol 7: 76, 2014.

30. Wang J, Xu G, Shen F and Kang Y: miR-132 targeting cyclin E1 suppresses cell proliferation in osteosarcoma cells. Tumour Biol 35: 4859-4865, 2014.

31. Liu J, Gu J, Feng Z, Yang Y, Zhu N, Lu W and Qi F: Both HDAC5 and HDAC6 are required for the proliferation and metastasis of melanoma cells. J Transl Med 14: 7, 2016.

32. Wang XC, Ma Y, Meng PS, Han JL, Yu HY and Bi LJ: miR-433 inhibits oral squamous cell carcinoma (OSCC) cell growth and metastasis by targeting HDAC6. Oral Oncol 51: 674-682, 2015.

33. Guo DL, Zhang J, Yuen ST, Tsui WY, Chan AS, Ho C, Ji J, Leung SY and Chen X: Reduced expression of EphB2 that parallels invasion and metastasis in colorectal tumours. Carcinogenesis 27: 454-464, 2006

34. Oshima T, Akaike M, Yoshihara K, Shiozawa M, Yamamoto N, Sato T, Akihito N, Nagano Y, Fujii S, Kunisaki C, et al: Overexpression of EphA4 gene and reduced expression of EphB2 gene correlates with liver metastasis in colorectal cancer. Int J Oncol 33: 573-577, 2008.

35. Feng X, Wu Z, Wu Y, Hankey W, Prior TW, Li L, Ganju RK, Shen $\mathrm{R}$ and Zou X: Cdc25A regulates matrix metalloprotease 1 through Foxo1 and mediates metastasis of breast cancer cells. Mol Cell Biol 31: 3457-3471, 2011.

36. Zurita M, Lara PC, del Moral R, Torres B, Linares-Fernández JL, Arrabal SR, Martínez-Galán J, Oliver FJ and Ruiz de Almodóvar JM: Hypermethylated 14-3-3- $\sigma$ and ESRI gene promoters in serum as candidate biomarkers for the diagnosis and treatment efficacy of breast cancer metastasis. BMC Cancer 10: 217, 2010.

37. Sonaglio V, de Carvalho AC, Toledo SR, Salinas-Souza C, Carvalho AL, Petrilli AS, de Camargo B and Vettore AL: Aberrant DNA methylation of ESRI and p14ARF genes could be useful as prognostic indicators in osteosarcoma. Onco Targets Ther 6: 713-723, 2013. 